\title{
Review Article Exploring Cortical Plasticity and Oscillatory Brain Dynamics via Transcranial Magnetic Stimulation and Resting-State Electroencephalogram
}

Submitted: 21 Apr 2016

Accepted: 21 Apr 2016

Online: 30 June 2016

\author{
Nor Azila $\mathbf{N o H}$
}

Department of Medical Science I, Faculty of Medicine and Health Sciences, Universiti Sains Islam Malaysia, Pandan Indah, 55100 Kuala Lumpur, Malaysia

To cite this article: Noh NA, Exploring cortical plasticity and oscillatory brain dynamics via transcranial magnetic stimulation and resting-state electroencephalogram. Malays J Med Sci. 2016; 23(4): 5-16. doi: 10.21315/ mjms2016.23.4.2

To link to this article: http://dx.doi.org/10.21315/mjms2016.23.4.2

\begin{abstract}
Transcranial magnetic stimulation (TMS) is a non-invasive, non-pharmacological technique that is able to modulate cortical activity beyond the stimulation period. The residual aftereffects are akin to the plasticity mechanism of the brain and suggest the potential use of TMS for therapy. For years, TMS has been shown to transiently improve symptoms of neuropsychiatric disorders, but the underlying neural correlates remain elusive. Recently, there is evidence that altered connectivity of brain network dynamics is the mechanism underlying symptoms of various neuropsychiatric illnesses. By combining TMS and electroencephalography (EEG), the functional connectivity patterns among brain regions, and the causal link between function or behaviour and a specific brain region can be determined. Nonetheless, the brain network connectivity are highly complex and involve the dynamics interplay among multitude of brain regions. In this review article, we present previous TMS-EEG co-registration studies, which explore the functional connectivity patterns of human cerebral cortex. We argue the possibilities of neural correlates of long-term potentiation/ depression (LTP-/LTD)-like mechanisms of synaptic plasticity that drive the TMS aftereffects as shown by the dissociation between EEG and motor evoked potentials (MEP) cortical output. Here, we also explore alternative explanations that drive the EEG oscillatory modulations post TMS. The precise knowledge of the neurophysiological mechanisms underlying TMS will help characterise disturbances in oscillatory patterns, and the altered functional connectivity in neuropsychiatric illnesses.
\end{abstract}

Keywords: brain stimulation, long-term potentiation (LTP), long-term depression (LTD), oscillations, plasticity

\section{Introduction}

Plasticity is defined as the ability of the brain to reorganise itself in order to primarily improve the functioning of the brain networks (1). Plasticity enables the modification of neural interaction that outlasts the experimental manipulation. Artificial brain stimulation such as transcranial magnetic stimulation can perturb the cortical and network oscillations (2). The residual effects of TMS are not confined to the stimulated cortex, but may spread across the functionally connected cortical circuits, such as the corticocortical and the cortico-thalamic networks (3). This functional connectivity can be explored using electroencephalogram through the modulation of brain oscillatory activity (4).

The hypotheses that suggest a link between the residual effects of TMS and cortical plasticity are due to the ability of TMS to induce changes that outlast the period of stimulation (4). The plasticity-like effect of TMS implies the potential use of this artificial non-invasive magnetic stimulation for basic neurophysiology research as well as for rehabilitation and therapy. It was proposed that TMS interferes with both neuronal and non-neuronal processes (5). The neuronal mechanisms range from local cellular changes to global-scale alteration of neuronal circuits such as network oscillations (6). The cellular changes 
consist of the local synaptic processes of synaptic excitation, synaptic inhibition, and synaptic plasticity, which are akin to the mechanisms of plasticity of long-term potentiation and longterm depression in animal studies (7). Other neuronal changes involve neuromodulators such as dopamine, growth factors such as brainderived neurotrophic factor (BDNF), and early genes proteins (8). In addition to neuronal effects, TMS also alters non-neuronal processes including cerebral blood flow by changes in blood oxygen level dependent (BOLD) and brain metabolic activity such as glucose metabolism [8]. However, it has always been assumed that the mechanism underlying cortical plasticity is the driving force of sustained TMS aftereffects. Unfortunately, the precise mechanism of TMSinduced cortical plasticity particularly in humans remains elusive. The ability of TMS to emulate the patterns of synaptic plasticity in the hippocampus suggests that TMS can affect synaptic efficacy in the neural network and modulate the cortical and network oscillatory activity (2). However, the cortical and network changes induced by TMS are still relatively unknown. Cortical neurons are largely interconnected and consist of various neural networks ranging from the simple, microlevel interconnections, to the dynamic and complex macro-level networks (5). The neural networks are widely implicated in the process of cortical information coding. Network oscillations through the balance of synchronisation and desynchronisation of neural assemblies are the important mechanisms involved during cortical information transfer (5). However, knowledge on the cortical and network oscillatory activity is still limited.

There is increasing evidence that has demonstrated the link between abnormal electrophysiological properties of network oscillations and the generation of neurological and psychiatric disorders (9). Altered brain rhythms are seen in patients of Parkinson's disease, schizophrenia, epilepsy, neurophatic pain, tinnitus, migraines, major depression, obsessivecompulsive disorder and psychosis. The term "Thalamocortical dysrhythmia (TCD)" describes abnormal prolonged low-frequency oscillations of delta and theta brain rhythms seen in patients of various neurological and psychiatric disorders (10). Although low-frequency oscillations are normal during slow-wave sleep, prolonged slow oscillations, such as theta rhythms, during awake periods and at rest interrupt the complex dynamic flow of information between the thalamus and cortex, and therefore may produce symptoms of neuropsychiatric illnesses (10). The combined TMS-EEG could have wide applicability in clinical research for characterising disturbances in oscillatory patterns and the altered functional connectivity in neuropsychiatric illnesses [9]. By directly entrain the oscillatory brain rhythms in a control manner, the TMS-EEG can indeed offer exciting possibilities as a diagnostic and therapeutic tool (11). Despite the rise in clinical research exploring the therapeutic potential of TMS, and the evidence of altered brain rhythms in neuropsychiatric patients, knowledge of the precise mechanisms of cortical oscillatory activity after the full range of TMS applications is still lacking.

In this review paper, we discuss the neurophysiological mechanisms of TMS aftereffects on cortical and network oscillations in humans. Focusing on EEG as the direct index of cortical output, the goal was to develop a deeper understanding of the modulation of oscillatory activity through various brain rhythms after noninvasive magnetic stimulation. In particular, we discuss what EEG response patterns may emulate LTP-/LTD-like changes that lead to corresponding changes in brain excitability and also provide information on versatile mechanisms of TMS actions, possibly exploitable in therapy.

\section{Transcranial Magnetic Stimulation}

In 1985, Professor Anthony Barker and colleagues from the University of Sheffield, UK, introduced transcranial magnetic stimulation (12). TMS is a non-invasive, non-pharmacological neurophysiologic method of delivering electrical stimuli by rapidly changing the magnetic field (12). TMS induces current into the brain without physical contact, as there are no implanted or surface electrodes. Instead, it works by placing an electromagnetic coil that carries pulses of current near the human scalp. The current from the TMS coil will generate an intense but brief magnetic field (up to 2 Tesla that lasts for $100 \mu$ s) that passes through the scalp, skull, and meninges to the cortical region beneath the coil without attenuation (13). Based on Faraday's law of electromagnetic induction, the rapidly changing magnetic field will induce an electrical current in the surrounding cortical tissue below the coil. As body tissue is electrically conductive, the ionic current will flow, eliciting nerve depolarisation and action potentials, and will subsequently stimulate the cortical neurons (13).

TMS has several advantages. Firstly, TMS does not generate strong pain because the TMS 
coil is placed near the scalp with no actual physical contact (14). Therefore, the induced current of magnetic stimulation does not pass through the skin-the location of most pain nerve endingsresulting in minimal or no activation of the pain receptors. Secondly, the current induced by magnetic stimulation does not attenuate because of the relatively low frequency magnetic fields that pass through the scalp, skull and meninges [14]. Thirdly, the current induced by magnetic stimulation is more diffuse, therefore relatively safe, provided strict safety guidelines are followed (15). All these advantages make TMS the method of choice in studying the physiology of the living human brain.

\section{TMS-EEG Co-Registration}

EEG helps us to understand the activity of the cerebral cortex by measuring the generalised electrical activity of a large population of cortical neurons (16). In 1929, Hans Berger (1873-1941), a German neuropsychiatrist, measured the first EEG on the human scalp using ordinary radio equipment to amplify the brain's electrical activity. In the defining experiment, Berger demonstrated that weak electrical current generated in the cortex could be recorded non-invasively over the human scalp (16). Berger introduced the word "electroencephalogram" to describe the electrical activity of the brain recorded from the human scalp. However, in modern times, the term EEG also applies to the electrical activity generated by brain structures as measured directly from the cortical surface (electrocorticogram), or within the brain using depth electrodes both in humans and in animals (16).

In spite of the modern functional neuroimaging such as PET and fMRI, which are able to assess the functional states of the brain, the scalp EEG is still considered as an important research and diagnostic tool in neuroscience. The advantage of scalp EEG over functional neuroimaging is its high temporal resolution of less than a millisecond, which enables real-time brain behavioural analysis (17). Moreover, EEG can directly record brain electrical activity, it is relatively inexpensive, and is simple to record. The EEG signal does not come from action potentials of the cortical neurons but from the electrical potentials of the pyramidal dendrites of the cortex (17). The electrical potentials are generated by the summed inhibitory and excitatory postsynaptic potentials from the pyramidal neurons of the cerebral cortex that produce electrical dipoles between the soma and the apical dendrites. The synchronous inputs of a large population of neurons make EEG a sensitive tool to study interaction between cortical areas and the functional connectivity of cortical networks (18).

The interest in brain rhythms has paralleled the development of non-invasive brain stimulation, such as TMS, and the advancement of computational tools for the time-frequency analysis of oscillatory dynamics (19). The simultaneous measurements of synchronised combinations of TMS and EEG enable the investigator to stimulate brain circuits while simultaneously monitoring changes in brain activity (19). EEG has the temporal sensitivity that allows an investigation into the immediate effects of TMS and provides a means to study the instantaneous neuronal effects of TMS in the brain with a millisecond time scale, which is presently not possible with any functional imaging method, as blood flow responses usually take some seconds following changes in neuronal activity (20). Such a simultaneous approach provides the opportunity to investigate the local responses to TMS at a neurophysiological level, thus helping to determine the brain areas that are either directly or transynaptically affected by magnetic stimulation.

The applications of TMS-EEG co-registration can be classified into three categories: inductiveusing TMS-EEG as index of brain physiological state in behaviourally silent regions; interactiveusing TMS-EEG to investigate the functional and dynamics of the brain; rhythmic-using TMS-EEG to study the generation and functional significance of brain rhythms (21). The inductive approach of TMS-EEG uses TMS-evoked potentials (TEP) recorded over the scalp as markers of the internal state of the brain in behaviourally silent areas. The interactive approach of TMS-EEG investigations involves the application of these combined methods to explore the transient modulation of neuronal networks during task performance (20). This approach is mainly used to identify the cortical area that is involved in a particular task. The rhythmic approach uses TMS-EEG to examine the modulation of oscillatory brain activity by rTMS and the link between specific frequency bands and their functional role (21). The significance of this approach is the potential role of using TMS to transiently modify brain functions by altering brain oscillations, and therefore, it may contribute to the therapeutic strategy of using TMS to reverse abnormal synchronisation in neuropsychiatric disorders (22). 


\section{Alteration of Cortical Oscillations Induced By TMS}

The first study that demonstrated the ability of magnetic stimulation to alter cortical oscillatory activity was by Paus et al. (23). The authors delivered single-pulse TMS over the sensorimotor cortex at rest and showed increased synchronisation of beta band $(15-30 \mathrm{~Hz})$ that lasted for several hundred milliseconds. This brief increase of synchronisation reflects the ability of TMS to induce the resetting of oscillations in a "resting" brain (23). A follow-up study by Fuggetta et al. (24) showed that single-pulse TMS applied over M1 at rest induced synchronisation in the alpha and beta bands for $500 \mathrm{~ms}$ post magnetic stimulation, and increased linearly with stimulus intensity. As Paus et al. stated previously, the authors concluded that the TMSinduced oscillations were linked to the resetting of the cortical oscillators, instead of the "idling" state of the brain $(23,34)$.

In 2006, Van Der Werf and Paus investigated the oscillatory activity of patients with Parkinson's disease who underwent partial thalamotomyunilateral surgery of the ventrolateral nucleus of the thalamus (25). Applying TMS over the intact hemisphere, the authors observed higher synchronisation of beta frequency band in the unoperated hemisphere (with the thalamus intact) than in the operated hemisphere (with thalamotomy) (25). This result implies the role of the thalamus in generating cortical oscillatory activity through various cortico-cortical networks and cortico-thalamic feedback loops (26). However, the oscillating properties depend on the connectivity of different pacemakers and the modulation of the reticular system, which is interconnected with all the thalamic nuclei (26). Besides thalamus, basal ganglia have an important role in driving oscillatory activity in the human motor cortex during motor performance (27). Using TMS, it has been shown that beta frequencies is prominent during tonic contraction but is attenuated prior to and during voluntary movement (28). In Parkinson's disease, the alterations of basal ganglia physiology may involve the alteration in the pattern of neuronal synchronisation particularly involving beta brain rhythms (28). The level of beta synchronisation is in turn modulated by net dopamine levels at sites of cortical input to basal ganglia (29). Dopamine deficiency as in the case of Parkinson's disease will disrupt the cortico-basal ganglia-thalamocortical circuits, leading to pathologically exaggerated beta oscillations (29).
In recent years there has been a growing interest in the cortical oscillatory activity at "rest" as an index of the internal state of the brain (30). The term "rest" represents the cortex during behaviourally silent states, with the absence of any sensory or motor output (30). The properties of neuronal oscillatory brain rhythms in a resting brain can provide the baseline for researchers and clinicians in distinguishing the oscillatory patterns that may be disrupted in patients of various neuropsychiatric disorders. However, only few studies have examined the potential use of TMS to transiently modulate brain rhythms over primary motor cortex (M1) at "rest". These studies have shown that the response of the EEG oscillatory state of the sensorimotor cortex at "rest" depends on TMS intensity, frequency of magnetic stimulation, and the total number of magnetic pulses. Strens et al. (2002) applied a train of 1500 pulses of $1 \mathrm{~Hz}$ repetitive TMS (rTMS) for 25 minutes over M1 at a subthreshold intensity (31). They demonstrated a decrease in EEG power of $\alpha$ frequency band of $6 \%$ and a focal increase of coherence during active task compared to resting condition ipsilateral to the site of stimulation [31]. A follow up study by Oliviero et al. (32) used a short train of 50 pulses of high frequency $5 \mathrm{~Hz}$ rTMS over M1 at active motor threshold. They showed a significant decrease in cortico-cortical interhemispheric coherence in the upper alpha frequency band $(10.7-13.6 \mathrm{~Hz})$ between the motor and premotor cortex for a few minutes after magnetic stimulation (32). In an online rTMS-EEG study, Fuggetta et al. (2008) used spectral analysis of event-related power (ERPow) and event-related coherence (ERCoh) to reveal how intermittent short trains of high frequency $(5 \mathrm{~Hz})$ rTMS delivered over left M1 induced an ERPow increase in upper alpha $(10-12 \mathrm{~Hz})$ and beta $(18-22 \mathrm{~Hz})$ frequency ranges for threshold (100\% RMT) and subthreshold intensities (80\% RMT). ERCoh showed a decrease in functional coupling for subthreshold rTMS in alpha and threshold rTMS for beta band (33). However, the aftereffect of rTMS in this experiment was short lasting - confined to 50oms after the magnetic stimulation-with no effect found two seconds after the train of magnetic pulses (33). Another on-line rTMS-EEG study by Brignani et al. explored the immediate effects of low frequency $1 \mathrm{~Hz}$ rTMS on the ongoing cortical oscillatory activity at "rest" (34). They delivered $1 \mathrm{~Hz}$ rTMS over M1 at $110 \%$ AMT of 600 stimuli. They showed a simultaneous increase of synchronisation of $\alpha(8-12 \mathrm{~Hz})$ more than $\beta(12-3 \mathrm{OHz})$ across all three stimulation blocks, which was inversely 
correlated with the progressive decrease of MEP amplitude [34]. Veniero et al. investigated the effects of the ongoing oscillatory activity of M1 at rest after high frequency $20 \mathrm{~Hz}$ rTMS (35). They observed increased synchronisation in alpha $(8-12 \mathrm{~Hz})$ more than beta $(13-3 \mathrm{OHz})$, and alpha induction lasted for 5 -min after magnetic stimulation. They showed a dose dependent increase of synchronisation in both the alpha and beta activities, spreading from the central region to the posterior, parietal sites (35).

In most TMS studies involving humans, low and high stimulation frequencies often result in opposite physiological effects as index by motor evoked potentials (MEP), expressed as either an increase or decrease in the amplitude of MEP (20). Low stimulation frequency $(\leq 1 \mathrm{~Hz})$ decreases cortical excitability (MEP suppression) whereas high frequency stimulation $(\geq 1 \mathrm{~Hz})$ increases cortical excitability (MEP enhancement) (17). However, rTMS-EEG studies of low and high frequency protocols were not able to emulate the classical dichotomy between low versus high frequency rTMS of MEP measurements (35). Instead, they observed linear EEG synchronisation for both low and high frequency rTMS in both alpha and beta frequency bands (33, 34,35 ). The inability of rTMS-EEG to distinguish the opposite effect of low versus high frequency at the cortical level may be because alpha and beta frequency bands are not the best index to reflect the dichotomy between low versus high frequency. A differential effect of low-high frequency rTMS may be better demonstrated by the modulation of other brain rhythms such as theta oscillations (35).

Noh et al. investigated the short-term modulation of cortical oscillations after high frequency rTMS by manipulating the different number of magnetic pulses (36). They compared the cortical readout of direct electrophysiological EEG after high frequency rTMS $(\sim 11 \mathrm{~Hz})$ of 20 trains of 20 pulses (400 magnetic pulses; rTMS 20 ) versus 20 trains of 60 pulses (1600 magnetic pulses; rTMS 60) over the left M1 at rest. They found the distinctly different topography and temporal dynamics of theta and mu rhythms (36). The theta synchronisation was globally distributed across multiple locations of the EEG electrodes for 20 seconds after rTMS 60 pulses. The mu rhythm was focally distributed and dominated early for 5 seconds after rTMS 20 pulses. These findings point to the probable presence of independent theta and mu generators over the human motor network with different reactivity to rTMS (36). Fuggetta and Noh investigated whether low frequency brain rhythms such as delta and theta oscillations could be used to exhibit the dichotomy between the simple protocols of low and high frequency magnetic stimulation (9). Short trains of low frequency $1 \mathrm{~Hz}$ rTMS versus high frequency $5 \mathrm{~Hz}$ and $10 \mathrm{~Hz}$ rTMS over $\mathrm{M} 1$ at rest were applied with simultaneous EEG recordings (9). Their results showed the ability of low frequency EEG oscillations of delta and theta brain rhythms to contrast the modulatory effects of low and high frequency rTMS (9). The findings of these experiments on the short-lasting modulation of low frequency oscillations after rTMS suggest that short trains of rTMS were able to induce shortterm plasticity-like mechanisms over the motor cortex. Although the rTMS-induced short-term plasticity-like mechanisms are enough for basic neuroscience research, the short-lasting effects are not sufficient for clinical intervention.

In order to investigate whether TMS could modulate EEG oscillatory activity for relatively longer periods of time, a pattern rTMS protocol of continuous theta burst stimulation (cTBS) was used. cTBS has been shown to induce longer-lasting behavioural effects after magnetic stimulation (37). In this experiment, the authors applied 300 pulses of short intensity but high frequency cTBS over the left M1 and measured the EEG oscillatory activity both at rest and during an active motor task [38]. Their results showed that cTBS could modulate the cortical brain rhythms, particularly beta oscillations, for at least 30 minutes compared to the 20 minutes MEP suppression for both event-related power modulation (ERPow) and event-related coherence (ERCoh) (39). This finding suggests that EEG is probably a more sensitive index of cortical output after cTBS compared to MEP.

\section{Cortical Plasticity Induced By TMS}

The hypotheses that suggest a link between the residual effects of TMS and plasticity is due to the ability of TMS to induce changes that outlast the period of stimulation (5). The residual effects of TMS are thought to originate from synaptic plasticity because its effects tend to emulate the patterns of synaptic plasticity in the rodent hippocampus (5). The long-term changes in the strength of hippocampal synapses involve the mechanisms of long term potentiation (LTP) and long term depression (LTD), which describe the direction of a long-lasting change in synaptic strength (40). LTP is an increase in the synaptic efficacy that could last for hours, days or weeks following brief high-frequency stimulation 
(HFS) protocols (41). The HFS paradigm in animal experiments that can induce LTP in the hippocampus is a protocol that consists of a single train of $100 \mathrm{~Hz}$ for 1 second (100 pulses with 10 ms intervals) (41). Another HFS protocol is thetaburst stimulation that consists of 10 bursts (each burst is 4 pulses at $100 \mathrm{~Hz}$ ) that are separated by an interval of $200 \mathrm{~ms}$. LTD is the long lasting weakening of the strength of hippocampal synapses following low-frequency stimulation (LFS). The most frequent LFS protocol is a single train of $1 \mathrm{~Hz}$ for 10 minutes (600 pulses) or for 15 minutes (90o pulses) (41).

The cellular basis of LTP and LTD originates from the hippocampal synapses of the axons of CA3 neurons and the dendritic spines of CA1 pyramidal neurons (42). The CA3 axon terminals discharge glutamate while the CA1 neurons express three types of glutamatergic receptors: alphaamino-3-hydroxy-5-methyl-4-isoxazolepropionic acid receptor (AMPA-R), N-methyl-D-aspartate receptor (NMDA-R), and metabotropic glutamate receptor (mGluR). The induction of LTP depends on the influx of $\mathrm{Ca}^{2+}$ in the postsynaptic cell. It starts when glutamate binds to AMPA-R, allowing $\mathrm{Na}^{+}$to enter into the dendritic spine, resulting in membrane depolarisation (42). When the postsynaptic neuron is sufficiently depolarised, the $\mathrm{Mg}^{2+}$ ions that block the NMDA- $\mathrm{R}$ at resting membrane potential will be removed, thus opening the NMDA-R (43). As a result, $\mathrm{Ca}^{2+}$ enters the postsynaptic neuron, and activates calcium-sensitive signaling pathways such as calcium-calmodulin protein kinase II (CaMKII) that leads to phosphorylation and upregulation of the AMPA-R. HFS protocols during experimental stimulations are able to remove the $\mathrm{Mg}^{2+}$ block of the NMDA-R, probably because HFS activates many AMPA-R, thus eliciting alarge depolarisation in the dendritic spine. The mechanism of LTD induction also depends on NMDA-R activation, which elevates $\mathrm{Ca}^{2+}$ concentration postsynaptically [44]. The element that determines whether LTP or LTD is induced is the nature of the $\mathrm{Ca}^{2+}$ signal that activates specific pathways. Large and fast elevation of $\mathrm{Ca}^{2+}$ concentration induces LTP by activating CaMKII, whereas small and slow rises of $\mathrm{Ca}^{2+}$ induce LTD by activating protein phosphatases that leads to dephosphorylation and down-regulation of the AMPA-R (45). LTD can be induced by LFS protocols that will mildly stimulate NMDA-R and produce an intermediate elevation of $\mathrm{Ca}^{2+}$ concentration (45).

Several studies have attempted to extend the principles of synaptic plasticity in the animal investigations to the TMS alterations of human cortical excitability. Primary motor cortex has been the most investigated cortical region with regards to TMS-induced plasticity. The studies highlight the success of TMS protocols in emulating the induction paradigms for LTP and LTD by changes in MEP sizes that outlast the TMS application. One TMS protocols that is able to produce longterm changes that emulate the protocols used for inducing LTP and LTD in rodent preparations is theta burst stimulation. Huang et al. showed that two TBS modalities have opposite effects on motor cortex excitability reminiscent of LTP and LTD (37). The iTBS of 600 pulses at $80 \%$ AMT produce a facilitatory effect for 15 minutes, whereas cTBS of 300 or 600 pulses suppress MEP amplitude for 20 or 60 minutes, respectively (46). Other studies of iTBS and cTBS showed similar results. Several pharmacological studies of TBS in the human cortex revealed that NMDA receptors seem to have parallel roles in the plasticity of cortical synapses as in the hippocampus. In a double-blind placebocontrol study, Huang et al. (2007) investigated the residual effects of TBS by prescribing the NMDA-R antagonist memantine and measuring the MEP size (47). The authors discovered that memantine blocked both the facilitatory effect of iTBS and the suppressive effect of cTBS as shown in the difference of the MEP size compared to control. Other recent studies showed that TBS influences NMDA receptor activity in humans and thus provide evidence of the involvement of TMS in neuroplasticity (48).

Although the modulatory changes in cortical efficacy by rTMS seem to emulate the paradigms of synaptic plasticity, it is important to emphasis the difference between the plasticity studies of the animal hippocampus and TMS studies of the cerebral cortex. The excitation of neural tissue in animal studies of synaptic plasticity and TMS studies in humans is fundamentally different (45). The stimulation of hippocampal slices in LTP/LTD studies is focal, whereas rTMS stimulation has a larger spatial resolution ranging from $\mathrm{mm}^{2}$ to $\mathrm{cm}^{2}$. Moreover, the brain region stimulated by rTMS in human studies is the cerebral cortex, which has a structurally more complex network than the hippocampal circuits (46). Cortical neurons are placed in multi-layered arrangements (the canonical six layers), with abundant synaptic connections. Cortical neurons receive massive inputs from the thalamus and, in turn, project heavily to the same structure. Therefore, this suggests that TMS may affect the vast recursive loops of excitation and inhibition between the cortex and the thalamus, between the different areas of the cortex, and including loops 
of both cerebral hemispheres (48). Moreover, the majority of rTMS-induced plasticity studies in humans used MEP amplitudes, which represent an indirect index of plasticity at the neuronal level. In addition, MEP is a polysynaptic read-out, separated by at least three synapses from the TMS source, whereas LTP and LTD are monosynaptic events (48). Therefore, in order to obtain a more accurate interpretation, it is important to combine rTMS with a recording technique that is also linked by a single synapse to the TMS pulse. One such technique is high-density EEG, which can provide a monosynaptic cortical readout during and after magnetic stimulation (49).

Studies that combined TBS and EEG to investigate the effect of cortical excitability induced by magnetic stimulation are lacking (50). A study that examined EEG network oscillations post-cTBS of 600 pulses was performed on the frontal eye field of only four healthy subjects (51). The study demonstrated higher neuronal synchronisation of the cerebral hemisphere ipsilateral to the stimulation site relative to the non-stimulated hemisphere up to one hour with synchronisation computed for broadband EEG and all brain rhythms. The authors speculated that cTBS might interfere with information transfer through its effect on neuronal synchronisation (51). However, in their study, there was no direct comparison between surface EEG and behavioural measurements during rest and active conditions to look at post-cTBS cortical plasticity effects. Moreover, the authors changed the site of stimulation (frontal eye field instead of motor cortex), the stimulation intensity ( $80 \%$ RMT instead of $80 \%$ AMT), and modified the cTBS paradigm (30 Hz bursts repeated at $6 \mathrm{~Hz}$ ) from the original cTBS protocol introduced by
Huang et al. (2005), making direct comparison with the original protocol problematic (52). McAllister et al. investigated the modulation of cortical oscillatory activity by cTBS of 600 pulses after a visuomotor training task using both MEP and EEG measurements (53). The authors only found significant alpha power that was positively correlated with MEP after the visuomotor training. They concluded that EEG was not useful as an index of cortical output to plasticity-inducing paradigms such as cTBS. However, in that study, the EEG was recorded using a single electrode of $\mathrm{C}_{3}$ over the motor cortex, and was therefore unable to ascertain the possible cTBS effects on cortico-cortical coupling (53). An investigation using multi-channel EEG will provide a more thorough outlook on the effects of cTBS on the motor network excitability. In a subsequent experiment, Noh et al. addressed the lack of knowledge of cTBS effects on motor network oscillations and their correlation with behavioural measurements by applying the original cTBS protocol consisting of 100 bursts of three pulses (300 pulses) at 50Hz repeated every 200ms $(5 \mathrm{~Hz})$ in 13 healthy subjects and measured the EEG oscillatory properties using high-density multi-channel EEG (37). Their results showed that cTBS could modulate the cortical brain rhythms, particularly beta oscillations, for at least 30 minutes compared to the 20 minutes MEP suppression. This finding suggests that EEG is probably a more sensitive index of cortical output after cTBS compared to MEP (37).

In order to demonstrate the link between TMS and oscillatory brain dynamics and cortical plasticity, Table 1 summarises the TMS-EEG co-registration studies that explores the neural correlates. 
Table 1: TMS and EEG at rest

\begin{tabular}{|c|c|c|c|c|c|c|}
\hline $\begin{array}{l}\text { rTMS } \\
\text { Protocol }\end{array}$ & Study & Site & Intensity & $\begin{array}{l}\text { Number } \\
\text { of pulses }\end{array}$ & EEG measures & Notes \\
\hline rTMS $1 \mathrm{~Hz}$ & $\begin{array}{l}\text { Strens et al. } \\
(2002)\end{array}$ & M1 & $90 \%$ AMT & 1500 & ERCoh alpha & $\begin{array}{l}\text { Increase corticocortical \& } \\
\text { interhemispheric coherence } \\
\text { in alpha for } 25 \text {-min }\end{array}$ \\
\hline TMS $5 \mathrm{~Hz}$ & $\begin{array}{l}\text { Oliviero et } \\
\text { al. (2003) }\end{array}$ & M1 & $90 \%$ AMT & 50 & ERCoh alpha & $\begin{array}{l}\text { Decrease ipsilateral } \\
\text { corticocortical } \\
\text { intrahemispheric coherence } \\
\text { in upper alpha }\end{array}$ \\
\hline rTMS $1 \mathrm{~Hz}$ & $\begin{array}{l}\text { Brignani et } \\
\text { al. (2008) }\end{array}$ & M1 & $110 \%$ RMT & 600 & $\begin{array}{l}\text { ERPow for alpha } \\
\text { and beta }\end{array}$ & $\begin{array}{l}\text { Increase ERPow alpha for } 10 \\
\text { minutes inversely correlated } \\
\text { with MEP }\end{array}$ \\
\hline $\begin{array}{l}\text { rTMS } \\
5 \mathrm{~Hz}\end{array}$ & $\begin{array}{l}\text { Fuggetta et } \\
\text { al. (2008) }\end{array}$ & M1 & $\begin{array}{l}80 \% \text { vs. } \\
100 \% \text { RMT }\end{array}$ & 400 & $\begin{array}{l}\text { ERPow, ERCoh } \\
\text { upper alpha and } \\
\text { beta }\end{array}$ & $\begin{array}{l}\text { Increase ERPow alpha }> \\
\text { beta. Decrease ERCoh, effect } \\
<2 \mathrm{~s}\end{array}$ \\
\hline cTBS & $\begin{array}{l}\text { Schindler } \\
\text { et al. } \\
\text { (2008) }\end{array}$ & FEF & $80 \%$ AMT & $\begin{array}{l}600(3 \\
\text { pulses } \\
\text { at } 30 \mathrm{~Hz}, \\
\text { repeated } \\
\text { every } \\
\text { 10oms }\end{array}$ & $\begin{array}{l}\text { Spectral power } \\
\text { delta, theta, alpha, } \\
\text { beta, gamma, for } \\
\text { 6o-min }\end{array}$ & $\begin{array}{l}\text { EEG synchronisation for } \\
\text { stimulated hemisphere } \\
\text { relative to non-stimulated } \\
\text { hemisphere for all frequency } \\
\text { bands }\end{array}$ \\
\hline $\begin{array}{l}\text { rTMS } \\
2 \mathrm{OHz}\end{array}$ & $\begin{array}{l}\text { Veniero et } \\
\text { al. (2011) }\end{array}$ & M1 & $100 \% \mathrm{RMT}$ & 400 & $\begin{array}{l}\text { ERPow alpha and } \\
\text { beta }\end{array}$ & $\begin{array}{l}\text { Dose dependent increase } \\
\text { ERPow alpha }>\text { beta for } 5 \\
\text { minutes, inversely correlated } \\
\text { with MEP }\end{array}$ \\
\hline $\begin{array}{l}\text { rTMS } \\
11 \mathrm{~Hz}\end{array}$ & $\begin{array}{l}\text { Noh et al. } \\
\text { (2011) }\end{array}$ & M1 & $100 \% \mathrm{RMT}$ & $\begin{array}{l}400 \mathrm{vs} . \\
1200\end{array}$ & $\begin{array}{l}\text { ERPow and } \\
\text { ERCoh theta, mu } \\
\text { and beta }\end{array}$ & $\begin{array}{l}\text { Increase ERPow theta }>\text { mu } \\
>\text { beta for } 1200 \text { pulses }>400 \\
\text { pulses }\end{array}$ \\
\hline cTBS & $\begin{array}{l}\text { McAllister } \\
\text { et al. (2011) }\end{array}$ & M1 & 80\% AMT & $\begin{array}{l}600(3 \\
\text { pulses } \\
\text { at } 50 \mathrm{~Hz}, \\
\text { repeated } \\
\text { every } \\
\text { 20oms) } \\
\text { for } 40 \mathrm{Os}\end{array}$ & $\begin{array}{l}\text { Spectral power } \\
\text { for baseline delta, } \\
\text { theta, alpha, } \\
\text { beta after visuo- } \\
\text { training task }\end{array}$ & $\begin{array}{l}\text { No increase EEG } \\
\text { synchronisation at rest, } \\
\text { MEPs suppression, } \\
\text { increase EEG power } \alpha \text { after } \\
\text { visuomotor training task }\end{array}$ \\
\hline cTBS & $\begin{array}{l}\text { Noh et al. } \\
\text { (2012) }\end{array}$ & M1 & 80\% AMT & $\begin{array}{l}300(3 \\
\text { pulses } \\
\text { at } 50 \mathrm{~Hz}, \\
\text { repeated } \\
\text { every } \\
200 \mathrm{~ms}) \\
\text { for } 20 \mathrm{~s} \\
\end{array}$ & $\begin{array}{l}\text { ERD/ERS, ERPow } \\
\text { and ERCoh theta, } \\
\text { alpha, low beta, } \\
\text { high beta at rest } \\
\text { and active; MEP } \\
\text { at rest; reaction } \\
\text { time active }\end{array}$ & $\begin{array}{l}\text { EEG at rest, EEG active for } \\
30 \text {-min, MEP at rest, RT } \\
\text { active }\end{array}$ \\
\hline $\begin{array}{l}\text { rTMS } \\
1 \mathrm{~Hz}, 5 \mathrm{~Hz} \\
10 \mathrm{~Hz}\end{array}$ & $\begin{array}{l}\text { Noh et al. } \\
\text { (2013) }\end{array}$ & M1 & $100 \% \mathrm{RMT}$ & 400 & $\begin{array}{l}\text { ERPow theta, mu } \\
\text { and beta }\end{array}$ & $\begin{array}{l}\text { Increase ERPow theta }>\text { mu } \\
>\text { beta for } 20 \text { at rest }\end{array}$ \\
\hline $\begin{array}{l}\text { rTMS } \\
1 \mathrm{~Hz}, 10 \mathrm{~Hz}\end{array}$ & $\begin{array}{l}\text { Noh et al. } \\
(2015)\end{array}$ & M1 & $100 \% \mathrm{RMT}$ & 400 & $\begin{array}{l}\text { ERCoh delta, } \\
\text { theta, mu and beta }\end{array}$ & $\begin{array}{l}\text { Increase ERCoh delta }>\text { theta } \\
>\mathrm{mu}>\text { for } 20 \text { at rest }\end{array}$ \\
\hline
\end{tabular}




\section{Limitations and Suggestions for Future Research}

Although TMS-induced modulatory aftereffects share many similarities with the mechanisms of synaptic plasticity, the evidence for such associations is, however, indirect. Studies of combined TMS or neuroimaging techniques such as PET and fMRI, and electrophysiological techniques such as EEG and MEG, have found strong indirect links between TMS and plasticity, but direct evidence is still lacking. Animal studies can offer better flexibility in order to establish a direct link between rTMS and plasticity.

Moreover, synaptic plasticity is probably not the only mechanism underlying the residual effects of magnetic stimulation because the EEG oscillatory activity is not correlated with MEP amplitude. Although TMS and plasticity share many characteristics such as TMS has effects that outlast the experimental manipulation, the temporal pattern of TMS-the frequency dependency effects-is similar to LTP/LTD, TMS plays a role in learning, TMS directly impairs of facilitates LTP in rats, however there is no causal proof that the underlying mechanisms of LTP/ LTD and TMS are identical. It is more likely that there is a multiplicity of mechanisms driving the sustained TMS aftereffects (54). Alternative mechanisms driving the modulatory aftereffects of rTMS are altered membrane excitability due to the influence of membrane potentials and ion channels (2). The membrane potential is an important determinant of excitability. The response of a nerve to sequences of impulses at sub- or supra-threshold levels results in a time dependent pattern of excitability changes, which follows changing levels of depolarisation and hyperpolarisation at the axonal membrane. Other alternative mechanisms include reduced cortical excitability in the resting states, increased excitability at the spinal cord, and breakdown of cortical inhibition (19). Future studies should address the multiplicity of mechanisms that drive the rTMS aftereffects besides LTP-/LTD-like mechanisms.

The ability of rTMSto modulatelow frequency brain rhythms such as theta oscillations is an exciting phenomenon (36). Evidence from EEG and MEG studies demonstrate that the common link among a wide range of neuropsychiatric disorders is the perturbation of the thalamocortical resonance known as Thalamocortical dysrhythmia
(TCD) (9,10). The idea behind TCD is that persistent, abnormal, internally generated delta or theta oscillations in the thalamic neurons disrupt the normal, state-dependent, flow of information within the thalamo-corticothalamic network. Although the occurrence of low frequency oscillations is normal during slowwave sleep, during awake periods, and at rest, prolonged delta and theta rhythms may lead to the disturbances of sensation, motor performance and cognition observed in a number of disorders including Parkinson's disease, schizophrenia, epilepsy, neuropathic pain, tinnitus, major depression, and obsessive-compulsive disorder (55). In parallel, several TMS protocols have been shown to be able to improve symptoms of various neuropsychiatric disorders although the optimal parameters of magnetic stimulation remain elusive (56). However, to our knowledge, there is very limited studies that investigates the probable link between TMS aftereffects and the TCD phenomenon in clinical populations. Can rTMS reverse TCD, thus alleviating the numerous symptoms in neuropsychiatric disorders? Future clinical trials can exploit the ability of combined rTMS-EEG to modulate and measure the dysrhythmic thalamocortical oscillatory activity in neuropsychiatric disorders.

\section{Acknowledgements}

None

\section{Conflict of Interests}

None

\section{Funds}

None

\section{Correspondence}

Dr. Nor Azila Noh

MD (UKM), MMedSc (UM), PhD (University of Leicester)

Department of Medical Science I,

Faculty of Medicine and Health Sciences,

Universiti Sains Islam Malaysia,

Level 13, Menara B, Persiaran MPAJ,

Jalan Pandan Utama, Pandan Indah,

55100 Kuala Lumpur

Tel: $+603-42892400$

Fax: +603-42892477

E-mail: azila@usim.edu.my 


\section{References}

1. Chen R, Udupa K. Measurement and modulation of plasticity of the motor system in humans using transcranial magnetic stimulation. Motor Control. 2009;13(4):442-453.

2. Opitz A, Fox MD, Craddock RC, Colcombe S, Milham MP. An intergrated framework for trageting functional networks via transcranial magnetic stimulation. Neuroimage. 2016;127:89-96. doi: http://dx.doi. org/10.1016/j.neuroimage.2015.11.040.

3. Di Lazzaro V, Ziemann U, Lemon RN. State of the art: Physiology of transcranial motor cortex stimulation. Brain Stimul. 2008;1(4):345-362. doi: http:// dx.doi.org/10.1016/j.brs.2008.07.004

4. Hallett M, Rothwell J. Milestones in clinical neurophysiology. Mov Disord. 2011; 26(6):958-967. doi: http://dx.doi.org/10.1002/mds.23572

5. Huerta PT, Volpe BT. Transcranial magnetic stimulation, synaptic plasticity and network oscillations. J Neuroeng Rehabil. 2009; 6:6-7. doi: http://dx.doi.org/10.1186/1743-0003-6-7

6. Frohlich F. Experiments and models of cortical oscillations as a target for noninvasive brain stimulation. Prog Brain Res. 2015;222:41-73. doi: http://dx.doi.org/10.1016/bs.pbr.2015.07.025

7. Malenka RC, Bear MF. LTP and LTD: an embarrassment of riches. Neuron. 2004;44(1):5-21. doi: http://dx.doi.org/10.1016/j.neuron.2004.09.012

8. Wang HY, Crupi D, Liu J, Stucky A, Cruciata G, Di Rocco A, et al. Repetitive transcranial magnetic stimulation enhances BDNF-TrkB signaling in both brain and lymphocyte. $J$ Neurosci. 2011;31(30):11044-11054. doi: http://dx.doi. org/10.1523/JNEUROSCI.2125-11.2011

9. Fuggetta G, Noh NA. A neurophysiological insight into the potential link between transcranial magnetic stimulation, thalamocortical dysrhythmia and neuropsychiatric disorders. Exp. Neurol. 2013;245:87-95. doi: http://dx.doi.org/10.1016/j. expneurol.2012.10.010

10. Henning Proske J, Jeanmonod D, Verschure PFM. A computational model of thalamocortical dysrhythmia. The European $J$ of Neuroscience. 2011;33(7):12811290. doi: http://dx.doi.org/10.1111/j.14609568.2010.07588.x

11. Miniussi C, Thut G. Combining TMS and EEG offers new prospects in cognitive neuroscience. Brain Topogr. 2010; 22(4):249-256. doi: http://dx.doi. org/10.1007/s10548-009-0083-8

12. Barker AT, Jalinous R, Freeston IL. Non-invasive magnetic stimulation of human motor cortex. Lancet. 1985; 8437:1106-1107. doi: http://dx.doi. org/10.1016/So140-6736(85)92413-4

13. Hallett M. Transcranial magnetic stimulation: a primer. Neuron. 2007;55(2):187-199. doi: http:// dx.doi.org/10.1016/j.neuron.2007.06.026
14. Eldaief MD, Press DZ, Pascual-Leone A. Transcranial magnetic stimulation in neurology. Neurol Clin Pract. 2013;3(6):519-526. doi: http://dx.doi.org/10.1212/o 1.0000436213.11132.8e.

15. Rossi S, Hallett M, Rossini PM, Pascual-Leone A. Safety, ethical considerations, and application guidelines for the use of transcranial magnetic stimulation in clinical practice and research. Clin Neurophysiol. 2009;120(12):2008-2039. doi: http://dx.doi.org/10.1016/j.clinph.2009.08.016

16. Maki H, Ilmoniemi, RJ. EEG oscillations and magnetically evoked motor potentials reflect motor system excitability in overlapping neuronal populations. Clin Neurophysiol. 2010;121(4):492501. doi: http://dx.doi.org/10.1016/j.clinph.2009. 11.078

17. Manganotti P, Formaggio E, Storti SF, De Massari D, Zamboni A, Bertoldo A, et al. Time-frequency analysis of short-lasting modulation of EEG induced by intracortical and transcallosal paired TMS over motor areas. $J$ Neurophysiol. 2012;107(9):2475-2484. doi: http://dx.doi.org/10.1152/jn.00543.2011

18. Niedermeyer E. The clinical relevance of EEG interpretation. Clin Electroencephalogr. 2003; 34(3): 93-98. doi: http://dx.doi.org/10.1177/ 155005940303400303

19. Bergmann TO, Karabanov A, Hartwigsen G, Thielscher A, Siebner HR. Combining non-invasive transcranial brain stimulation with neuroimaging and electrophysiology: current approaches and future perspectives. NeuroImage. Forthcoming 2016; doi: http://dx.doi.org/10.1016/j.neuroimage.2016.02.012

20. Thut G, Veniero D, Romei V, Miniussi C, Schyns P, Gross J. Rhythmic TMS causes local entrainment of natural oscillatory signatures. Curr Biol. 2011;21(14):1176-1185. doi: http://dx.doi.org/10. 1016/j.cub.2011.05.049

21. Schyns PG, Thut G, Gross J. Cracking the code of oscillatory activity. PLoS Biol. 2011;9(5):e1001064. doi: http://dx.doi.org/10.1371/journal.pbio.1001064

22. Thut G, Schyns PG, Gross J. Entrainment of perceptually relevant brain oscillations by noninvasive rhythmic stimulation of the human brain. Front Psychol. 2011;2:170. doi: http://dx.doi. org/10.3389/fpsyg.2011.00170

23. Paus T, Sipila PK, Strafella AP. Synchronization of neuronal activity in the human primary motor cortex by transcranial magnetic stimulation: an EEG study. J Neurophysiol. 2001;86(4):1983-1990.

24. Fuggetta G, Fiaschi A, Manganotti P. Modulation of cortical oscillatory activities induced by varying singlepulse transcranial magnetic stimulation intensity over the left primary motor area: a combined EEG and TMS study. Neuroimage. 2005;27(4):896-908. doi: http://dx.doi.org/10.1016/j.neuroimage.2005.05.013

25. Van Der Werf YD, Paus T. The neural response to transcranial magnetic stimulation of the human motor cortex. Intracortical and cortico-cortical contributions. Exp Brain Res. 2006;175(2):231-245. doi: http://dx.doi.org/10.1007/s00221-006-0551-2 
26. Sherman, S. M. The thalamus is more than just a relay. Curr Opin Neurobiol. 2007;17(4):417-422. doi: http://dx.doi.org/10.1016/j.conb.2007.07.003

27. Haber SN, Calzavara R. The cortico-basal ganglia integrative network: the role of the thalamus. Brain Res Bull. 2009;78(2):69-74. doi: http://dx.doi. org/10.1016/j.brainresbull.2008.09.013

28. Chen R. Repetitive transcranial magnetic stimulation as treatment for depression in Parkinson's disease. Mov Disord. 2010;25(14):2272-2273. doi: http:// dx.doi.org/10.1002/mds.23266

29. Zanjani A, Zakzanis KK, Daskalakis ZJ, Chen R. Repetitive transcranial magnetic stimulation of the primary motor cortex in the treatment of motor signs in Parkinson's disease: A quantitative review of the literature. Mov Disord. 2015;30(6):750-758. doi: http://dx.doi.org/10.1002/mds.26206

30. Stamoulis C, Oberman LM, Praeg E, Bashir S, PascualLeone A. Single pulse TMS-induced modulations of resting brain neurodynamics encoded in EEG phase. Brain Topogr. 2011;24(2):105-113. doi: http:// dx.doi.org/10.1007/s10548-010-0169-3

31. Strens LH, Oliviero A, Bloem BR, Gerschlager W, Rothwell JC, Brown P. The effects of subthreshold $1 \mathrm{~Hz}$ repetitive TMS on cortico-cortical and interhemispheric coherence. Clin Neurophysiol. 2002;113(8):1279-1285. doi: http://dx.doi org/10.1016/S1388-2457(02)oo151-7

32. Oliviero A, Strens LH, Di Lazzaro V, Tonali PA, Brown P. Persistent effects of high frequency repetitive TMS on the coupling between motor areas in the human. Exp Brain Res. 2003;149(1):107-113.

33. Fuggetta G, Pavone EF, Fiaschi A, Manganotti P. Acute modulation of cortical oscillatory activities during short trains of high-frequency repetitive transcranial magnetic stimulation of the human motor cortex: a combined EEG and TMS study. Hum Brain Mapp. 2008;29(1):1-13. doi: http://dx.doi.org/10.1002/ hbm.20371

34. Brignani D, Manganotti P, Rossini PM, Miniussi C. Modulation of cortical oscillatory activity during transcranial magnetic stimulation. Hum Brain Mapp. 2008;29(5):603-612. doi: http://dx.doi. org/10.1002/hbm.20423

35. Veniero D, Brignani D, Thut G, Miniussi C. Alphageneration as basic response-signature to transcranial magnetic stimulation (TMS) targeting the human resting motor cortex: A TMS/EEG co-registration study. Psychophysiology. 2011;48(10):1381-1389. http://dx.doi.org/10.1111/j.1469-8986.2011.01218.x

36. Noh NA, Fuggetta G. Human cortical theta reactivity to high-frequency repetitive transcranial magnetic stimulation. Hum Brain Mapp. 2011;33(9):22242237.

37. Huang YZ, Edwards MJ, Rounis E, Bhatia KP Rothwell JC. Theta burst stimulation of the human motor cortex. Neuron. 2005;45(2):201-206. doi: http://dx.doi.org/10.1016/j.neuron.2004.12.033
38. Noh NA, Fuggetta G, Manganotti P, Fiaschi A. Long lasting modulation of cortical oscillations after continuous theta burst transcranial magnetic stimulation. PloSOne. 2012; doi: http://dx.doi. org/10.1371/journal.pone.0035080

39. Noh NA, Fuggetta G, Manganotti P. Theta burst transcranial magnetic stimulation alters the functional topography of the cortical motor network. Malays J Med Sci. 2015; Special Issue:36-44.

40. Malenka RC. Synaptic plasticity in the hippocampus: LTP and LTD. Cell. 1994;78(4):535-538. http:// dx.doi.org/10.1016/0092-8674(94)90517-7

41. Malenka RC. Synaptic plasticity and AMPA receptor trafficking. Ann N Y Acad Sci. 2003;1003:1-11. doi: http://dx.doi.org/10.1196/annals.1300.001

42. Paoletti P, Bellone C, Zhou Q. NMDA receptor subunit diversity: impact on receptor properties, synaptic plasticity and disease. Nature Rev Neurosc. 2013;14:383-400. doi: http://dx.doi.org/10.1038/ nrn3504

43. Teo JT, Swayne OB, Rothwell JC. Further evidence for NMDA- dependence of the aftereffects of human theta burst stimulation. Clin Neurophysiol. 2007;118(7):1649-1651. doi: http://dx.doi. org/10.1016/j.clinph.2007.04.010

44. Zhang Y, Llinas RR, Lisman JE. Inhibition of NMDARs in the Nucleus Reticularis of the Thalamus Produces Delta Frequency Bursting. Front Neural Circuits. 2009;3:20.

45. Ziemann U. LTP-like plasticity in human motor cortex. Suppl Clin Neurophysiol. 2004;57:702-707. doi: http://dx.doi.org/10.1016/S1567-424X(o9)70410-6

46. Huang YZ, Rothwell JC, Chen RS, Lu CS, Chuang WL. The theoretical model of theta burst form of repetitive transcranial magnetic stimulation. Clin Neurophysiol. 2011; 122(5):1011-1018. doi: http:// dx.doi.org/10.1016/j.clinph.2010.08.016

47. Huang YZ, Chen RS, Rothwell JC, Wen HY. The aftereffect of human theta burst stimulation is NMDA receptor dependent. Clin Neurophysiol. 2007;118(5):1028-1032. doi: http://dx.doi.org/10. 1016/j.clinph.2007.01.021

48. Huang YZ, Rothwell JC, Edwards MJ, Chen RS. Effect of physiological activity on an NMDAdependent form of cortical plasticity in human. Cereb Cortex. 2008;18(3):563-570. doi: http://dx.doi. org/10.1093/cercor/bhmo87

49. Hughes SW, Crunelli V. Just a phase they're going through: the complex interaction of intrinsic high-threshold bursting and gap junctions in the generation of thalamic alpha and theta rhythms. Int $J$ Psychophysiol. 2007;64(1):3-17. doi: http://dx.doi. org/10.1016/j.ijpsycho.2006.08.004

50. Ishikawa S, Matsunaga K, Nakanishi R, Kawahira K, Murayama N, Tsuji S, et al. Effect of theta burst stimulation over the human sensorimotor cortex on motor and somatosensory evoked potentials. Clin Neurophysiol. 2007;118(5):1033-1043. doi: http:// dx.doi.org/10.1016/j.clinph.2007.02.003 
51. Nyffeler T, Wurtz P, Luscher HR, Hes CW, Senn W, Pflugshaupt T, et al. Repetitive TMS over the human oculomotor cortex: comparison of $1-\mathrm{Hz}$ and theta burst stimulation. Neurosci Lett. 2006;409(1):57-60. doi: http://dx.doi.org/10.1016/j.neulet.2006.09.011

52. Grossheinrich N, Rau A, Pogarell O, Hennig-Fast K, Reinl M, Karch S, et al. Theta burst stimulation of the prefrontal cortex: safety and impact on cognition, mood, and resting electroencephalogram. Biol Psychiatry. 2009;65(9):778-784. doi: http:// dx.doi.org/10.1016/j.biopsych.2008.10.029

53. McAllister SM, Rothwell JC, Ridding MC. Cortical oscillatory activity and the induction of plasticity in the human motor cortex. Eur $J$ Neurosci. 2011;33(10):1916-1924. doi: http://dx.doi.org/ 10.1111/j.1460-9568.2011.07673.x
54. Choudhury K, Boyle L, Burke M, Lombard W, Ryan S, McNamara B. Intra subject variation and correlation of motor potentials evoked by transcranial magnetic stimulation. Ir J Med Sci. 2011;180(4):873-880. doi: http://dx.doi.org/10.1007/s11845-011-0722-4

55. Schulman JJ, Cancro R, Lowe S, Lu F, Walton, KD, Llinas RR. Imaging of thalamocortical dysrhythmia in neuropsychiatry. Front in human neurosc. 2011; 5:1-11. doi: http://dx.doi.org/10.3389/ fnhum.2011.00069

56. Kimiskidis VK. Transcranial magnetic stimulation (TMS) coupled with electroencephalography (EEG): biomarker of the future. Rev Neurol. 2016;172(2):123-126. doi: http://dx.doi.org/10. 1016/j.neurol.2015.11.004 